This is a pre-copyedited, author-produced PDF of an article accepted for publication in Behavioral Ecology following peer review. The version of record Claudia Santori, Luc F Bussière, Thomas M Houslay, Heightened perception of competition hastens courtship, Rehavioral Ecology, Volume 31, Jssue 1, January/February 2020, Pages 239-246, is available online at: https://doi.org/10.1093/beheco/arz183

\title{
1 Heightened perception of competition hastens courtship
}

2 Claudia Santori ${ }^{1,2}$, Luc F Bussière ${ }^{1}$, Thomas M Houslay ${ }^{1,3 *}$

3

$4 \quad{ }^{1}$ University of Stirling, Stirling, FK9 4LA, UK

$5 \quad 2$ Current address: School of Life and Environmental Sciences, The University of

6 Sydney, Camperdown, NSW 2050, Australia

$7 \quad{ }^{3}$ Current address: Department of Zoology, University of Cambridge, Downing

8 Street, Cambridge, CB2 3EJ, UK

$9 *$ Corresponding author

10 Email: $\underline{\text { houslay@gmail.com }}$

11

12 Short title: Competition hastens courtship

13

14 


\section{Abstract}

16 When animals use costly labile display or signal traits to display to the opposite

17 sex, they face complex decisions regarding the degree and timing of their

18 investment in separate instances of trait expression. Such decisions may be

19 informed by not only the focal individual's condition (or pool of available

20 resources), but also aspects of the social environment, such as perceptions of

21 same-sex competition or the quality of available mates. However, the relative

22 importance of these factors to investment decisions remains unclear. Here we

23 use manipulations of condition (through dietary nutrition), recent social

24 environment (exposure to a silenced male, non-silenced male, female, or

25 isolation), and female mating history (single- or multiple-male) to test how

26 quickly male decorated crickets (Gryllodes sigillatus) decide to begin courting an

27 available female. We find that males that were previously housed with non-

28 silenced males started courting the female earlier than other males. Females only

29 mounted males after courtship began. Our results suggest a strong effect of the

30 perception of competition on the decision to invest resources in sexual signalling

31 behaviour, and that females might exert directional selection on its timing.

32

33 Keywords: Sexual selection, courtship, condition-dependence, social experience,

34 sexual signalling, phenotypic flexibility, Gryllodes sigillatus. 
37 Sexual signalling displays are some of the most extravagant and diverse traits observed in nature, and both sexual selection and life history theory inform us as to their evolution and consequences. Investment in mate attraction displays often confers a much higher mating success, but at some cost to their bearer (Darwin 1871; Andersson 1994). A ubiquitous cost of sexual trait investment is simply that any individual has only a finite pool of resources that can be allocated to its various traits. Thus, investment in one trait also represents a loss

44 of potential investment in all other competing traits. Individuals that can acquire more resources have a greater pool from which to allocate, meaning the marginal costs of additional investment in condition-dependent displays should be lower (Van Noordwijk and De Jong 1986). This may be why males in high condition tend to spend more on mate attraction (Hunt et al. 2004), and experimental manipulations of diet have resulted in increased signalling or display effort in a variety of taxa (e.g., fiddler crabs Uca beebei, Backwell et al. 1995; wolf spiders Hygrolycosa rubrofasciata, Kotiaho 2000; field crickets Gryllus campestris, Holzer et al. 2003).

53

54 In species where individuals express their sexual trait repeatedly across their

55 lifetime, resource-based trade-offs occur between not only the focal trait and

56 other components of life history, but also current and future expression of the

57 focal trait. Given that resources invested at one stage are unavailable for

58 investment at another, individual condition is therefore critical in determining 
59 both the intensity of male signalling and the most suitable allocation pattern of

60 current versus future reproductive effort (Bretman et al. 2011).

62 If the signal trait in question is a behavioural display, the resolution of trade-offs can be highly dynamic and responsive to short-term changes in the local environment (Bretman et al. 2011). The ability of individuals to respond plastically to immediate changes in the local environment should be selected for in species living in very unpredictable habitats, or where mating success may be highly dependent on the number of mating rivals and mating opportunities. Social cues influencing male behaviour can range from the population sex ratio and density, to encounters and matings with females (Bateman and Fleming 2006; Bailey et al. 2010; Bretman et al. 2011). However, the importance of the social environment relative to other factors affecting plastic reproductive effort (e.g., diet or mate quality) remains unclear. Furthermore, tracking one’s own status and predicting fitness is likely quite complicated in natural environments (Kasumovic et al. 2012). Simple behavioural rules (e.g., "spend resources if you have them, and if not, focus on acquiring resources instead"; Houslay et al., 2017) may be more likely than complex adaptive plasticity to explain variation in social, age, and status-dependent reproductive effort.

In this study we quantify how resource acquisition and cues of the local social environment influence the timing and intensity of sexual trait investment in the decorated cricket, Gryllodes sigillatus. Male crickets signal to females using stridulation of their hardened forewings, through which they can produce two types of calls: a long-range call to attract females from far away, and a close- 
range courtship-call just before mating (Ketola et al. 2007). Time spent signalling (typically referred to as 'calling effort') is a strong predictor of mating success in nature (Hunt et al. 2004; Bentsen et al. 2006; Rodriguez-Munoz et al. 2010), but is energetically expensive (e.g., Kavanagh 1987; Hunt et al. 2004; Ophir et al. 2010; Mowles 2014) and may increase mortality risk from both intrinsic (Hunt et al. 2004) and extrinsic sources (Cade 1975; Walker 1979). Previous work on

\section{G. sigillatus has shown that increased dietary nutrition leads to an increase in} both the likelihood and amount of signalling in early adulthood, as well as to greater investment in energy stores (Houslay et al. 2017). The same study demonstrated that signalling investment is highly responsive to the availability of a potential mate: males are more likely to signal, and signal for longer, if a female is present relative to when absent. In related species of crickets, the recent or current presence of rival males at adulthood can increase calling effort (Callander et al. 2013; Noguera 2018), suggesting plasticity of signalling behaviour based on perceived competition. Manipulations of the juvenile social environment have also indicated that crickets can perceive future competition rates and adjust investment in reproductive tissues (Bailey et al. 2010) and agespecific calling effort (Kasumovic et al. 2013) accordingly.

Here we manipulate resource acquisition ability in males from the day of eclosion to adulthood using diets that vary in nutritional content. We then use a 2-day 'social environment' manipulation, providing cues of future competition or mating opportunities by exposing males to either another male, a female, or keeping them isolated. We monitor their calling effort during this period to estimate their immediate response to the social situation. We then provide each 
male access to a female cricket and observe their latency to begin courtship. To assess the importance of female reproductive value in determining courtship

111 speed, female crickets were all mated previously, having been given the

112 opportunity to mate with either only a single male or multiple males.

114 We hypothesise that male G. sigillatus housed with other males may perceive a

115 greater level of competition relative to isolated males, and should thus begin

116 courtship more quickly when next encountering a female. Males who are

117 exposed to a female during the social environment manipulation instead might

118 perceive greater mating opportunities and low competition, and thus begin

119 courting later. We predict that increased dietary nutrition level should decrease

120 the latency to call, as males with a greater pool of resources have less incentive

121 to conserve resources. We also hypothesise that males may start courting

122 females with only one previous partner more quickly than those with prior

123 access to multiple potential partners, as females with multiple previous mates

124 may present heightened levels of sperm competition in this highly polyandrous

125 species (Sakaluk 1987). G. sigillatus appear able to detect previous mating

126 partners using cuticular hydrocarbons (CHCs) that are transferred during

127 mating, and that vary according to both sex and genotype (Ivy et al. 2005;

128 Weddle, Mitchell et al. 2012; Weddle, Steiger et al. 2012). We expect a positive

129 relationship between male courtship latency and female mounting latency, such

130 that female $G$. sigillatus are likely to more quickly mount males that begin

131 courting earlier. Females may also prefer males on higher-quality diets as they

132 should be in better condition, and multiple-male females might be less keen to

133 mate than their single-male counterparts (based on the observation that female 
receptivity tends to decrease somewhat after the first mating, even for highly polyandrous species; Jennions and Petrie 2000; Wedell 2005; Judge et al. 2010).

\section{Methods}

138 Study species and mating behaviour

139 The decorated cricket (G. sigillatus) is probably native to South Asia, but is

140 common to tropical and subtropical regions worldwide (Otte 2006). Females

141 respond phonotactically to calling songs of conspecifics (Champagnon and Cueva

142 del Castillo 2008). Mating involves a female mounting the male in order to attach

143 his spermatophore (comprising a sperm ampulla and gelatinous

144 spermatophylax) to her (Alexander and Otte 1967), and forced copulations are 145 not possible in this species. The spermatophore comprises a sperm ampulla 146 surrounded by a spermatophylax (Sakaluk 1987; Ivy and Sakaluk 2005), which is 147 a gelatinous mass that the female separates from the ampulla and feeds on while 148 the sperm is transferred into her sperm receptacle (Sakaluk 1987). After 149 finishing eating or discarding the spermatophylax, the female removes the 150 ampulla too, therefore terminating the transfer of sperm. Females have a high 151 remating rate (Sakaluk 1987), and polyandry improves survival prospects of 152 offspring (Ivy and Sakaluk 2005). Males have a lower maximal mating frequency 153 than females (Sakaluk 1987), apparently due to the time required to build a new 154 spermatophore (Sakaluk 1985). The potential nutritional benefits to females of 155 the spermatophylax are controversial (Will and Sakaluk 1994; Warwick et al. 156 2009), although spermatophylax consumption provides a water-stressed female 157 with great hydration (Ivy et al. 1999), possibly representing one of the 158 advantages of mating multiply. 
160 Cricket rearing and maintenance

161 Experimental $G$. sigillatus were the $55^{\text {th }}$ generation of a laboratory stock

162 composed by 5000 individuals originally from Las Cruces, New Mexico, USA. The

163 previous generations were allowed to breed freely, with periodic new

164 introductions from cultures of other research institutions. The crickets used in

165 this study were born and maintained at $31 \pm 1{ }^{\circ} \mathrm{C}$ on a $14: 10 \mathrm{hr}$ light:dark

166 photoperiod in a controlled-temperature room set for lights off at 2:30 PM. We

167 reared the newly hatched nymphs in clear plastic containers $(30 \times 30 \times 15 \mathrm{~cm})$,

168 each containing several dozen individuals of the same age born from mixed

169 parents. We provided cricket nymphs with ad libitum water in $47 \mathrm{~mL}$ vials

170 stoppered with dampened cotton wool, ground dry cat-food (Friskies Go-Cat

171 Senior, Purina ${ }^{\circledR}$, London, UK), and egg cartons for shelter. We cleaned the

172 containers and replaced food and water weekly throughout the experimental

173 period. Males and females were reared together until the day of their eclosion, at

174 a density of approximately 200-300 crickets per container.

176 Experimental design and manipulations

177 We checked all nymphs every morning, collecting any individuals that had

178 eclosed to adulthood overnight. On the day of its eclosion we weighed every

179 individual with an electronic balance (PI-225DA, Denver Instrument, Bohemia,

$180 \mathrm{NY}$ ). We isolated each new adult male individually in a small plastic container (7

$181 \times 7 \times 7 \mathrm{~cm}$ ). These containers were supplied with $7 \mathrm{~mL}$ water vials plugged with

182 cotton wool, and plastic mesh attached to the sides of the container as substrate.

183 To manipulate the crickets' nutritional condition, we haphazardly assigned each 
adult male to one of five dry and granular artificial diets differing in energy content. All the diets had a 1:8 ratio of protein:carbohydrate, and the total protein and carbohydrate content of the food mass ranged from $36 \%$ to $84 \%$ of (the rest being a mix of vitamins and indigestible crystalline cellulose). Previous studies have shown that these diets affect individual condition and allocation to competing traits in male G. sigillatus (Rapkin et al. 2016; Houslay et al. 2017).

The experimental crickets had access to their assigned diet ad libitum for 10 days, until the behavioural trials. We measured body mass again at the end of the first adult week (day 7) in order to test for effect of diet treatment on any change in body mass.

\section{Social experience manipulation}

From their day of eclosion onwards, we isolated individuals acoustically by placing their containers into cubes of acoustic foam (Houslay et al. 2015; Houslay et al. 2017). Each foam lid had a small opening to allow light from the chamber inside the box, and we tested that this opening did not allow cross-talk from other cricket containers to contaminate our estimate of calling effort for a focal male (see below). At seven days post-eclosion (by which time males have greatly increased both the likelihood and intensity of calling effort; Houslay et al., 2017), we exposed males to one of four treatments designed to manipulate their social experience for a period of 2 days (i.e., days 8 and 9 post-eclosion): 1) control (maintained in isolation); 2) housed with a female; 3 ) housed with a silenced male (wings clipped); or 4) housed with an injured male as a sham, which was subject to autotomy of a single limb (a common escape mechanism in Orthopterans; Bateman and Fleming, 2008) that did not affect calling ability. The 
sham treatment was used instead of an unmanipulated treatment to account for

210 the potential effect of injury on male calling. We were unsure if wing clipping (for

211 silenced males) might reduce the activity of crickets, and whether any such

212 reduced activity might be perceptible to focal males. Since the sham treatment

213 was meant to reflect differences in calling attributable to calling by the non-focal

214 male, we wanted that male to have been similarly handled (and injured) in a way

215 that mirrored the silenced male, but without directly affecting calling. The social

216 partners of focal males were introduced to the experimental crickets' individual

217 containers inside pierced $60 \mathrm{~mL}$ transparent plastic vials containing some

218 soaked cotton for water and a pellet of commercial cat food. Tactile contact

219 between crickets was possible only through the holes pierced on the sides of the

220 vials, which were not large enough to allow mating. The crickets for this social

221 manipulation were stock individuals of the same generation, but not taking part

222 in the experiment.

224 We recorded calling effort over both nights of the social manipulation

225 experiment by inserting a microphone (C1163, Dick Smith Electronics) into the

226 lid of each individual male container, which we connected to an Electronically

227 Activated Recorder (EAR; Bertram and Johnson 1998). The EAR samples each

228 microphone 10 times per second to determine whether the assigned cricket was

229 calling or not. We started recording every day as the lights went out (2:30 PM)

230 and stopped the following day (9:30 AM). On the morning of day 9 post-eclosion,

231 we ended the social experience manipulation, weighing the experimental males

232 and returning them to their original container for a day of isolation before the

233 courtship behaviour trials. 
235 Females mating history manipulation

236 After collecting their morphological measures upon eclosion, we housed females 237 of similar age together in a $30 \times 30 \times 15 \mathrm{~cm}$ plastic container. We provided them 238 with ground dry cat food and water ad libitum until day 7 post-eclosion. At day 7 , 239 we randomly assigned each female to one of two treatment groups: single-male 240 (SM) and multiple-males (MM). We placed a female assigned to the SM treatment 241 in a $7 \times 7 \times 7 \mathrm{~cm}$ plastic container with food and water, one stock male, and 242 moistened cotton on a petri dish as oviposition substrate. We placed a female 243 assigned to the MM treatment in a bigger plastic container $(12 \times 12 \times 12 \mathrm{~cm})$, 244 containing up to three females and many stock males (3-4 males for every 245 female), as well as food, water and oviposition substrate. We left each female in 246 either of these groups for 2 days, after which we weighed her and placed her in 247 isolation for one day in a $7 \times 7 \times 7 \mathrm{~cm}$ container supplied with food and water. 248 The following day, we performed the courtship behavioural trials. SM and MM 249 treatments therefore differ in potential mating frequency and probable number 250 of mates; however, we did not verify mating frequency for individual females.

251 Nevertheless, it is very likely that in two days there would be more than one 252 mating due to G. sigillatus females' typical mating frequency (Sakaluk 1987).

254 Mating behaviour trials

255 At 10 days post-eclosion we randomly paired one experimental male with either 256 an SM or MM female, using a no-choice experimental paradigm (e.g., Shackleton 257 et al. 2005; Judge et al. 2010). All the mating trials took place soon after the main 258 lights went out, under illumination from a $25 \mathrm{~W}$ red incandescent bulb held 
about $40 \mathrm{~cm}$ from the cricket containers to minimise any possible visual

disturbance. The female was introduced to the male in his individual container,

261 which was supplied with water but no food. We noted the time elapsed until the

262 male's first call (latency to call), as well as how long the female took to mount the

263 male after his courtship started (latency to mount). Each trial lasted a maximum

264 of 30 minutes, after which we ended the observation regardless of the state of

265 courtship.

267 Statistical analysis

268 We performed all analyses using R version 3.4.2 (R Core Team 2017), with the

269 'tidyverse' set of packages for data cleaning and visualisation (Wickham 2017).

270 For normally distributed response variables, we used generalised linear mixed

271 effects models (GLMMs) with restricted maximum likelihood (REML)

272 approaches in lme4 (Bates et al. 2015). In lme4, we checked model fit visually

273 through diagnostic plots, and used parametric bootstrapping (with 1000

274 simulations) to assess the difference between nested models refitted with ML for

275 hypothesis testing (R package pbkrtest; Halekoh and Højsgaard 2014). For

276 overdispersed count data (see below) we used Bayesian estimation in

277 MCMCglmm (Hadfield 2010). Here we checked model fit visually through plots of

278 MCMC chains for both variance components and fixed effects, in addition to

279 testing that multiple runs converged to similar results via the Gelman-Rubin

280 diagnostic (Gelman \& Rubin 1992) and that models were robust to different

281 priors. We used 95\% credible intervals of posterior distributions for hypothesis

282 testing in these models. For pairwise comparison of groups within categorical

283 predictors, we subtracted the posterior distribution of one group from another, 
and inspected the $95 \%$ credible interval of the resulting 'difference' distribution

285 (such that if 0 is excluded then the difference between those groups is nominally 286 significant). In all models, the effect of diet was centred and scaled to single unit 287 increments (i.e., the 5 diets were treated as a continuous sequence from -2 to 2 ). 288 The 'social manipulation' and 'female mating status' manipulations were treated 289 as 4- and 2-level categorical variables respectively.

291 We assessed the effect of the nutritional manipulation using a mixed effects 292 model fit in lme4, where our response variable was 'body mass' and predictors 293 were diet, time period ( 0 and 1 to reflect start and end of first week post 294 eclosion), and their interaction. We also included a random effect of male cricket 295 ID. A significant positive interaction between time period and diet would 296 indicate that males on diets containing greater nutritional content increased 297 body mass at a higher rate over the course of the week.

299 Calling effort data during the social experience manipulation roughly 300 approximated a Poisson distribution and was highly overdispersed, so we 301 elected to use Bayesian methods, as MCMCglmm includes a vector of residuals 302 that handles overdispersion. Unlike previous studies of calling effort in this 303 species (e.g., Houslay et al. 2015; Houslay et al. 2017), the level of zero-inflation 304 was fairly low (less than 15\%) and so we used the overdispersed Poisson 305 distribution rather than a more specialist hurdle or zero-altered model. Our 306 model included fixed effects of the social manipulation, diet and their interaction, 307 day of observation (mean-centred) as a further covariate, and a random effect of 308 male ID. 
310 We also used overdispersed Poisson models fit in MCMCglmm to analyse the

311 effects of our treatments on (i) latency to begin courtship, and (ii) latency to

312 mount. For courtship latency, predictors included diet, social manipulation

313 treatments, and female mating status. As data were right-censored, the latency to

314 call was set to the maximum value (1800s) for males that did not call $(49 / 217)$.

315 For mounting latency, we excluded those 49 males that did not call, and used the

316 same predictors as above (male diet, male social manipulation treatments, and

317 female mating status) with the addition of log-transformed call latency (mean-

318 centred and scaled to standard deviation units after the log-transformation) as a

319 further predictor. Data were right-censored, so we set the latency to mount to 320 the maximum value (1800s) for females that did not mount the courting male $321(43 / 168)$

\section{Results}

324 A total of 217 male crickets completed the experiment and were included in our

325 final data set. Our experimental design resulted in 40 diet $\times$ social environment $\times$

326 female status combinations, each cell of which contained a minimum of 3 and

327 maximum of 11 individuals after excluding those that died during the

328 experiment (see Table S1 for full breakdown of sample size by experimental

329 manipulations).

331 Males tended to gain body mass over the course of the first week post-eclosion

332 on average, and mass increase was greater in those with access to diets of higher

333 nutritional content (parametric bootstrap P < 0.001; Figure 1, Table S2). Calling 
effort was higher in all treatments relative to control, although only significantly so in the two male treatments (Figure 2, Table 1). We found no interaction between diet and the manipulations of social environment (95\% CIs were large and centred close to zero for each term), and so refit the model excluding this interaction. Excluding the interaction had negligible effect on the coefficients for the main effects. Increased nutritional content tended to increase the level of calling effort, but this was not significant. We found that males decreased calling effort from the first day to the second of the social manipulation experiment. Table 1: Coefficients and 95\% credible intervals for the analysis of calling effort. Social manipulation treatment levels show deviations from the reference group ('isolated male').

\begin{tabular}{|l|r|r|r|}
\hline Parameter & Estimate & $95 \%$ CI & pMCMC \\
\hline (Intercept) & 3.42 & $2.66,4.13$ & $<0.001$ \\
\hline Diet & 0.21 & $-0.07,0.47$ & 0.14 \\
\hline Social: Female & 0.33 & $-0.63,1.31$ & 0.49 \\
\hline Social: Silenced male & 1.15 & $0.08,2.22$ & 0.04 \\
\hline Social: Sham male & 1.39 & $0.31,2.48$ & 0.01 \\
\hline Day & -0.72 & $-1.08,-0.29$ & 0.002 \\
\hline
\end{tabular}

The only significant predictor of the latency to call in the courtship behaviour trials was the 'sham male' treatment group, during which the focal male had been housed with a male that was able to call (Table 2). These males show a 
351 marked decrease in the time taken to begin courting the available female,

352 relative to isolated males (Figure 3). Previous housing with a silenced male also

353 tended to decrease latency to call, although the effect size was smaller and the

$35495 \%$ credible intervals did extend beyond zero (95\% CI: -1.22, 0.04; Table 2).

355 Dietary nutrition tended to reduce call latency, but again the credible intervals

356 (just) included zero (95\% CI: $-0.33,0.01$; Table 2).

357

358 Pairwise comparisons of treatment groups indicated that the sham male

359 treatment group had a significantly shorter latency to begin calling relative to the

360 female treatment group (95\% CI: $-1.62,-0.26)$. There was no significant

361 difference between sham male and silenced male treatment groups (95\% CI: -

$3621.14,0.28)$, nor between silenced male and female treatment groups (95\% CI: -

$3631.15,0.17)$ 
Table 2: Coefficients and 95\% credible intervals for the analysis of courtship latency. Higher values indicate increased time for the focal male to begin courting the available female in the behavioural trial. Categorical variables show deviations from the reference group (isolated for social manipulation; single male for female mating status).

\begin{tabular}{|l|r|r|r|}
\hline Parameter & Estimate & $95 \%$ CI & pMCMC \\
\hline (Intercept) & 4.94 & $4.40,5.46$ & $<0.001$ \\
\hline Diet & -0.16 & $-0.33,0.01$ & 0.07 \\
\hline Social: Female & -0.11 & $-0.74,0.51$ & 0.73 \\
\hline Social: Silenced male & -0.60 & $-1.22,0.04$ & 0.06 \\
\hline Social: Sham male & -1.08 & $-1.77,-0.42$ & 0.003 \\
\hline Mating: Multiple-males & 0.08 & $-0.37,0.59$ & 0.73 \\
\hline
\end{tabular}

370

372 We found no significant effects of our experimental manipulations on female

373 decision time (Table 3). Females mounted males only after courtship had been 374 initiated (Figure 4). 
Table 3: Coefficients and 95\% credible intervals for the analysis of mounting latency. Higher values indicate increased time for the female to mount the male in the behavioural trial. Categorical variables show deviations from the reference group (isolated for social manipulation; singly mated for female mating status).

\begin{tabular}{|l|r|r|r|}
\hline Parameter & Estimate & $95 \%$ CI & pMCMC \\
\hline (Intercept) & 5.02 & $4.47,5.64$ & $<0.001$ \\
\hline Diet & -0.08 & $-0.26,0.10$ & 0.40 \\
\hline Social: Female & 0.37 & $-0.36,1.02$ & 0.30 \\
\hline Social: Silenced male & 0.16 & $-0.50,0.89$ & 0.66 \\
\hline Social: Sham male & 0.28 & $-0.42,0.95$ & 0.41 \\
\hline Mating: Multiple-males & 0.46 & $-0.04,0.96$ & 0.09 \\
\hline
\end{tabular}

\section{Discussion}

383 A male's decision to invest in sexual signalling may be informed by both his energy budget and his experience of the social environment. Few studies have manipulated an animal's resource acquisition and social experience simultaneously, to assess their relative importance in mating interactions. Here, we find that the recent social environment plays a large role (seemingly larger than that of diet) in determining how quickly male G. sigillatus begin courting an

389 available female. Males that were housed recently with another male began 390 courting the female earlier, although this effect was strongest when the other 391 male was not silenced. The idea that male calling behaviour is strongly 
influenced by the perception of competition is reinforced by our observations of

393 calling effort during the social environment manipulation, where males that

394 were housed with other males (whether silenced or not) appear to call more

395 than males housed with females or by themselves. Our results suggest that male

396 crickets show both immediate and lasting (at least in the short-term)

397 behavioural plasticity, based on their experience of the social environment. The

398 importance of this effect is highlighted by the consequence of courtship latency

399 on mating, as females mounted males only after they commenced calling.

401 Exposure to rival males increases calling effort

402 Previous studies have shown that male calling effort is strongly affected by the 403 social environment, whether that be developmental plasticity caused by the 404 perception of future competition at the juvenile stage (Kasumovic et al. 2012; 405 Kasumovic et al. 2013), flexibility caused by recent or current exposure to rival males (Callander et al. 2013; Noguera 2018), or current access to potential mates 407 (Houslay et al. 2017). Here we show that males also exhibit an increase in calling 408 effort when exposed directly to another male. This does not appear to be due 409 solely to some 'chorus effect' (i.e., being provoked into calling via the calling of a 410 competitor), as we saw an increase in calling effort among males exposed to 411 silenced (i.e., wings removed) males as well as to the 'sham' injured males. We

412 note that while a sham male may be contributing to the observed calling effort 413 assigned to a focal male, in the silenced male treatment all calling must be from 414 the focal male. While this result indicates males do respond to perceived 415 competitors, males placed with females did not call more than those kept in 416 isolation - a result seemingly at odds with those of Houslay et al. (2017), in 
417 which exposure to female crickets greatly increased both the likelihood and

418 amount of male calling effort. However, males in our current study did not have

419 full physical access to the other individual (instead being separated by a plastic

420 barrier, albeit with holes to allow some degree of contact and airflow). As

421 posited by Houslay et al. (in whose study males had full physical access to

422 females and were able to mate), the positive feedback from females may drive

423 the increased calling by males. We note also that the calling effort recorded in

424 that study was far beyond that which was seen in ours, despite a similar nightly

425 recording period and similar dietary regimen. An open question concerns how

426 males were distinguishing differences in social environment in our study, which

427 could be based on visual or auditory, touch, or chemosensory (via CHC) cues.

Exposure to rival males decreases courtship latency

430 We originally hypothesised that males might be able to use a mechanism such as

431 information from CHCs to infer a female's number of previous matings, thus

432 enabling discrimination against females in the multiple-male treatment group

433 (who would likely present a higher intensity of sperm competition (Sakaluk

434 1986) and/or higher likelihood of carrying sexually transmitted nematodes

435 (Luong et al. 2006)). However, even if this is possible, we found no difference in

436 how quickly males started to court a female. We also found no effect of the

437 'female exposure' social manipulation on courtship latency. While males

438 exposed to females previously took longer to begin courting an available female

439 in the courtship trials relative to those exposed previously to males, there was no

440 difference between those exposed to females and those held in isolation.

441 Therefore, these results do not support our a priori hypothesis that the exposure 
to a female might alter behaviour due to a male's perception of mate availability,

443 causing a decrease in his signalling effort and urge to start courting the next

444 female encountered.

446 We do, however, see a strong response in courtship latency as a result of a male's

447 own prior exposure to other males, particularly those that were not silenced (i.e.,

448 the 'sham' male treatment). Combined with the strong increase in calling effort

449 during the social environment manipulation for males exposed to sham and

450 silenced rivals, our results suggest a strong and lasting effect of a short-term

451 change in the competitive social environment in this species. This effect held

452 despite a day spent in isolation between the social treatment and the mating

453 trial. Previous studies have shown that manipulations of the juvenile social

454 environment (using recordings of males played to mimic different densities)

455 induce developmental plasticity that affects how males invest in calling at

456 adulthood in a related species of cricket (Teleogryllus commodus; Kasumovic et

457 al. 2012; Kasumovic et al. 2013). Our results add support to the notion that male

458 crickets are highly tuned to their social environment, and likely use multiple

459 sources (including acoustic and chemical) to gather information regarding

460 potential competition for mating opportunities.

Mounting latency is related to calling latency

463 Females that had had access to multiple potential mates prior to the mating trials

464 showed only a small and non-significant increase in mounting latency,

465 suggesting that availability of multiple males previously did not greatly diminish

466 a female's receptivity. This result is in line with previous work in this species 
indicating that female $G$. sigillatus have a high re-mating rate, averaging 22 times every 20 days (Sakaluk 1987). Despite the lack of evidence for substantial

469 nutritional benefits of the spermatophylax (Will and Sakaluk 1994), previous

470 work has indicated that such a high re-mating rate may be offsetting any costs of 471 reproduction via some benefits of nuptial gifts provided by males (Burpee and 472 Sakaluk 1993). In our experiment, around 75\% of males that started courting in

473 trials were mounted by the female during their behavioural trial, with similar 474 proportions of callers mounted across single- and multiple-male females: 18/78 $475(77 \%)$ and 25/90 (72\%) respectively. This high female re-mating rate could be 476 selecting for responsiveness to mating opportunities in males, who would 477 benefit from advertising their availability as quickly as possible. These results 478 are also in line with patterns found by Houslay et al. (2017): over the course of a 479 week of continued access to females there was a decrease in calling effort (which 480 appeared due largely to declining energy reserves), but not of the likelihood of 481 calling. This pattern suggests that males have a strong inclination to court 482 females, even if they are in lower condition. We note that the dietary nutrition 483 manipulation used in the study of Houslay et al. (2017) did not show a 484 statistically significant effect of diet on the likelihood of calling, and here we find 485 a small and non-significant decrease in calling latency due to dietary nutrition.

487 Concluding remarks

488 Overall, our results show that male energy reserves tended to increase sexual 489 signalling duration and hasten the decision to start courting an available female, 490 but these effects were fairly weak and not statistically significant. Variation in 491 male signalling effort can be driven strongly by variation in the current or recent 
competitive environment, suggesting that males are gathering information from various sources to determine their behaviour. Our results provide further evidence for the flexibility of sexual signalling behaviour, which in turn suggests

495 that a male's ability to respond to current opportunities has been shaped by 496 substantial past selection. Additional investigation of how individuals gather 497 information and make decisions to outcompete their rivals and take advantage of 498 potential mating opportunities - and how this affects patterns of age-dependent 499 variation, as well as allocation to competing life history traits - might be a 500 fruitful avenue of research. More broadly, the field would benefit from more 501 quantitative assessments of the relative importance of multiple contributing 502 factors to behavioural variation.

\section{Funding}

505 This work was supported by the University of Stirling.

507 Acknowledgements

508 We are grateful to J. Weir and in particular P. Monteith for technical support, and

509 to J. Rapkin for making the diets used in this study. We also thank the Bussière

510 lab (especially E. Herridge and R. Murray) and various cricket luminaries

511 (especially J. Hunt) for discussion. Thanks also to L. Holman and 2 anonymous

512 reviewers for constructive comments on our manuscript.

514 Data Accessibility

515 Analyses reported in this article can be reproduced using the data provided by 516 Santori et al. (2019). 


\section{7}

\section{8}

519

520

521

522

523

524

535 Bentsen CL, Hunt J, Jennions MD, Brooks RC. 2006. Complex Multivariate Sexual

536 Selection on Male Acoustic Signaling in a Wild Population of Teleogryllus

537 commodus. Am. Nat. 167:102-116.

538 Bertram SM, Johnson L. 1998. An electronic technique for monitoring the

539 temporal aspects of acoustic signals of captive organisms. Bioacoustics 9:107-

540118.

541 Bretman A, Gage MJG, Chapman T. 2011. Quick-change artists: male plastic 
542 behavioural responses to rivals. Trends Ecol. Evol. 26:467-73.

543 Burpee DM, Sakaluk SK. 1993. Repeated matings offset costs of reproduction in

544 female crickets. Evol. Ecol. 7:240-250.

545 Cade W. 1975. Acoustically Orienting Parasitoids: Fly Phonotaxis to Cricket Song.

546 Science. 190:1312-1313.

547 Callander S, Kahn AT, Hunt J, Backwell PRY, Jennions MD. 2013. The effect of

548 competitors on calling effort and life span in male field crickets. Behav. Ecol.

$549 \quad 24: 1251-1259$.

550 Champagnon J, Cueva del Castillo R. 2008. Female Mate Choice, Calling Song and

551 Genetic Variance in the Cricket, Gryllodes sigillatus. Ethology 114:223-230.

552 Darwin C. 1871. The descent of man, and selection in relation to sex. London:

553 John Murray.

554 Hadfield JD. 2010. MCMC Methods for Multi-Response Generalized Mixed

555 Models: The MCMCglmm R Package. J. Stat. Softw. 33:1-25.

556 Halekoh U, Højsgaard S. 2014. A Kenward-Roger Approximation and Parametric

557 Bootstrap Methods for Tests in Linear Mixed Models - the R Package pbkrtest. J.

558 Stat. Softw. 59.

559 Holzer B, Jacot A, Brinkhof MWG. 2003. Condition-dependent signaling affects

560 male sexual attractiveness in field crickets, Gryllus campestris. Behav. Ecol.

$561 \quad 14: 353-359$.

562 Houslay TM, Houslay KF, Rapkin J, Hunt J, Bussière LF. 2017. Mating

563 opportunities and energetic constraints drive variation in age-dependent sexual

564 signalling. Miller C, editor. Funct. Ecol. 31:728-741.

565 Houslay TM, Hunt J, Tinsley MC, Bussière LF. 2015. Sex differences in the effects

566 of juvenile and adult diet on age-dependent reproductive effort. J. Evol. Biol. 
568 Hunt J, Brooks RC, Jennions MD, Smith MJ, Bentsen CL, Bussière LF. 2004. High-

569 quality male field crickets invest heavily in sexual display but die young. Nature $570 \quad 432: 1024-7$.

571 Ivy TM, Johnson JC, Sakaluk SK. 1999. Hydration benefits to courtship feeding in 572 crickets. Proc. R. Soc. B Biol. Sci. 266:1523-1528.

573 Ivy TM, Sakaluk SK. 2005. Polyandry promotes enhanced offspring survival in 574 decorated crickets. Evolution. 59:152-9.

575 Ivy TM, Weddle CB, Sakaluk SK. 2005. Females use self-referent cues to avoid 576 mating with previous mates. Proc. Biol. Sci. 272:2475-8.

577 Jennions MD, Petrie M. 2000. Why do females mate multiply? A review of the 578 genetic benefits. Biol. Rev. Camb. Philos. Soc. 75:21-64.

579 Judge KA, Tran K-C, Gwynne DT. 2010. The relative effects of mating status and 580 age on the mating behaviour of female field crickets. Can. J. Zool. 88:219-223.

581 Kasumovic MM, Hall MD, Brooks RC. 2012. The juvenile social environment 582 introduces variation in the choice and expression of sexually selected traits. Ecol. 583 Evol.

584 Kasumovic MM, Hall MD, Try H, Brooks RC. 2013. Socially cued developmental 585 plasticity affects condition-dependent trait expression. Behav. Ecol. 24:429-434. 586 Kavanagh MW. 1987. The efficiency of sound production in two cricket species, 587 Gryllotalpa australis and Teleogryllus commodus (Orthoptera: Grylloidea). J. Exp. 588 Biol. 130:107-119.

589 Ketola T, Kortet R, Kotiaho JS. 2007. Testing theories of sexual selection in 590 decorated crickets (Gryllodes sigillatus). Evol. Ecol. Res. 9:869-885.

591 Kotiaho JS. 2000. Testing the assumptions of conditional handicap theory: costs 
and condition dependence of a sexually selected trait. Behav. Ecol. Sociobiol. 48:188-194.

Luong LT, Platzer EG, Zuk M, Giblin-Davis RM. 2006. Venereal Worms: Sexually

595 Transmitted Nematodes in the Decorated Cricket. J. Parasitol. 86:471.

Mowles SL. 2014. The physiological cost of courtship: field cricket song results in anaerobic metabolism. Anim. Behav. 89:39-43.

598 Noguera JC. 2018. Crickets increase sexual signalling and sperm protection but 599 live shorter in the presence of rivals. J. Evol. Biol.:1-10.

600 Van Noordwijk AJ, De Jong G. 1986. Acquisition and allocation of resources: their

601 influence on variation in life history tactics. Am. Nat. 128:137-142.

602 Ophir AG, Schrader SB, Gillooly JF. 2010. Energetic cost of calling: general 603 constraints and species-specific differences. J. Evol. Biol. 23:1564-9.

604 Otte D. 2006. Gryllodes sigillatus (Walker) is a Valid Species Distinct from 605 Gryllodes supplicans (Walker). Trans. Am. Entomol. Soc. 132:223-227.

606 R Core Team. 2017. R: A language and environment for statistical computing. 607 Rapkin J, Jensen K, Lane SM, House CM, Sakaluk SK, Hunt J. 2016. Macronutrient 608 intake regulates sexual conflict in decorated crickets. J. Evol. Biol. 29:395-406.

609 Rodriguez-Munoz R, Bretman A, Slate J, Walling CA, Tregenza T. 2010. Natural 610 and Sexual Selection in a Wild Insect Population. Science. 328:1269-1272.

611 Santori C, Bussière LF, Houslay TM. 2019. Data from: Heightened perception of

612 competition hastens courtship. Behavioral Ecology.

613 https://doi.org/10.5061/dryad.h18931zfn

614 Sakaluk SK. 1985. Spermatophore size and its role in the reproductive behaviour 615 of the cricket, Gryllodes supplicans (Orthoptera: Gryllidae). Can. J. Zool. 63:16526161656. 
617 Sakaluk SK. 1986. Sperm competition and the evolution of nuptial feeding

618 behaviour in the cricket, Gryllodes supplicans (Walker). Evolution. 40:584-593.

619 Sakaluk SK. 1987. Reproductive behaviour of the decorated cricket, Gryllodes

620 supplicans (Orthoptera: Gryllidae): calling schedules, spatial distribution, and

621 mating. Behaviour 100:202-225.

622 Shackleton M., Jennions MD, Hunt J. 2005. Fighting success and attractiveness as

623 predictors of male mating success in the black field cricket, Teleogryllus

624 commodus: the effectiveness of no-choice tests. Behav. Ecol. Sociobiol. 58:1-8.

625 Walker TJ. 1979. Calling crickets (Anurogryllus arboreus) over pitfalls: Females,

626 males, and predators. Environ. Entomol. 8:441-443.

627 Warwick S, Vahed K, Raubenheimer D, Simpson SJ. 2009. Free amino acids as

628 phagostimulants in cricket nuptial gifts: support for the "Candymaker"

629 hypothesis. Biol. Lett. 5:194-6.

630 Weddle CB, Mitchell C, Bay SK, Sakaluk SK, Hunt J. 2012. Sex-specific genotype-

631 by-environment interactions for cuticular hydrocarbon expression in decorated

632 crickets, Gryllodes sigillatus: implications for the evolution of signal reliability. J.

633 Evol. Biol. 25:2112-2125.

634 Weddle CB, Steiger S, Hamaker CG, Ower GD, Mitchell C, Sakaluk SK, Hunt J. 2013.

635 Cuticular hydrocarbons as a basis for chemosensory self-referencing in crickets:

636 a potentially universal mechanism facilitating polyandry in insects. Grether G,

637 editor. Ecol. Lett. 16:346-353.

638 Wedell N. 2005. Female receptivity in butterflies and moths. J. Exp. Biol.

$639208: 3433-3440$.

640 Wickham H. 2017. tidyverse: Easily Install and Load “Tidyverse” Packages.

641 Will MW, Sakaluk SK. 1994. Courtship feeding in decorated crickets - is the 
642 spermatophylax a sham? Anim. Behav. 48:1309-1315.

643

644 


\section{Figure legends}

646 Figure 1: The effect of dietary nutritional content on the change in body mass

647 over the first week post-eclosion. Dark points and vertical bars show raw means

648 and standard errors; light points show individual data (jittered on x-axis with a

649 small amount of random noise). The horizontal dotted line at 0 indicates no

650 change in body mass.

Figure 2: The effect of social environment manipulation on calling effort during the treatment period. The 'isolation' treatment represents our control treatment

654 for analysis. Grey points are raw data (lightly jittered on x axis with small

655 amount of random noise); black points and line ranges show estimates and 95\% credible intervals from MCMCglmm analysis (averaging over effects of day and 657 diet), plotted on log scale.

Figure 3: Effects of the social environment manipulation on latency to call in the courtship behavioural trials. Males in the 'sham male' treatment group show a

661 significant reduction in call latency relative to isolated males. Grey points are 662 raw data; black points and line ranges are estimates and associated 95\% credible 663 intervals from MCMCglmm analysis, plotted on log scale. Note that males 664 assigned a censored score of $1800 \mathrm{~s}$ did not call before the end of the trial period.

666 Figure 4: Males were only mounted after they started actively courting females, 667 shown by the positive relationship between (log-transformed) latency to male 668 calling (x-axis) and (log-transformed) total time to mating (i.e., calling latency + 669 mounting latency; y-axis). Open circles indicate observations where males called 
670 but were not mounted by the female (and were assigned a censored score of

$6711800 \mathrm{~s}$ for latency to mount). Dotted line shows the 1:1 relationship between

672 latency to call and total time to mating. Males that did not call were not mounted,

673 and are not shown.

674

675 Supplementary material

676

677 Table S1: Sample sizes of males completing the experiment across each

678 combination of social manipulation, female mating status and dietary nutrition.

679

680 Table S2: Summary of mixed model (fitted in lme4 with Gaussian error family)

681 for analysis of the change in body mass over the first week post-eclosion.

682

683 


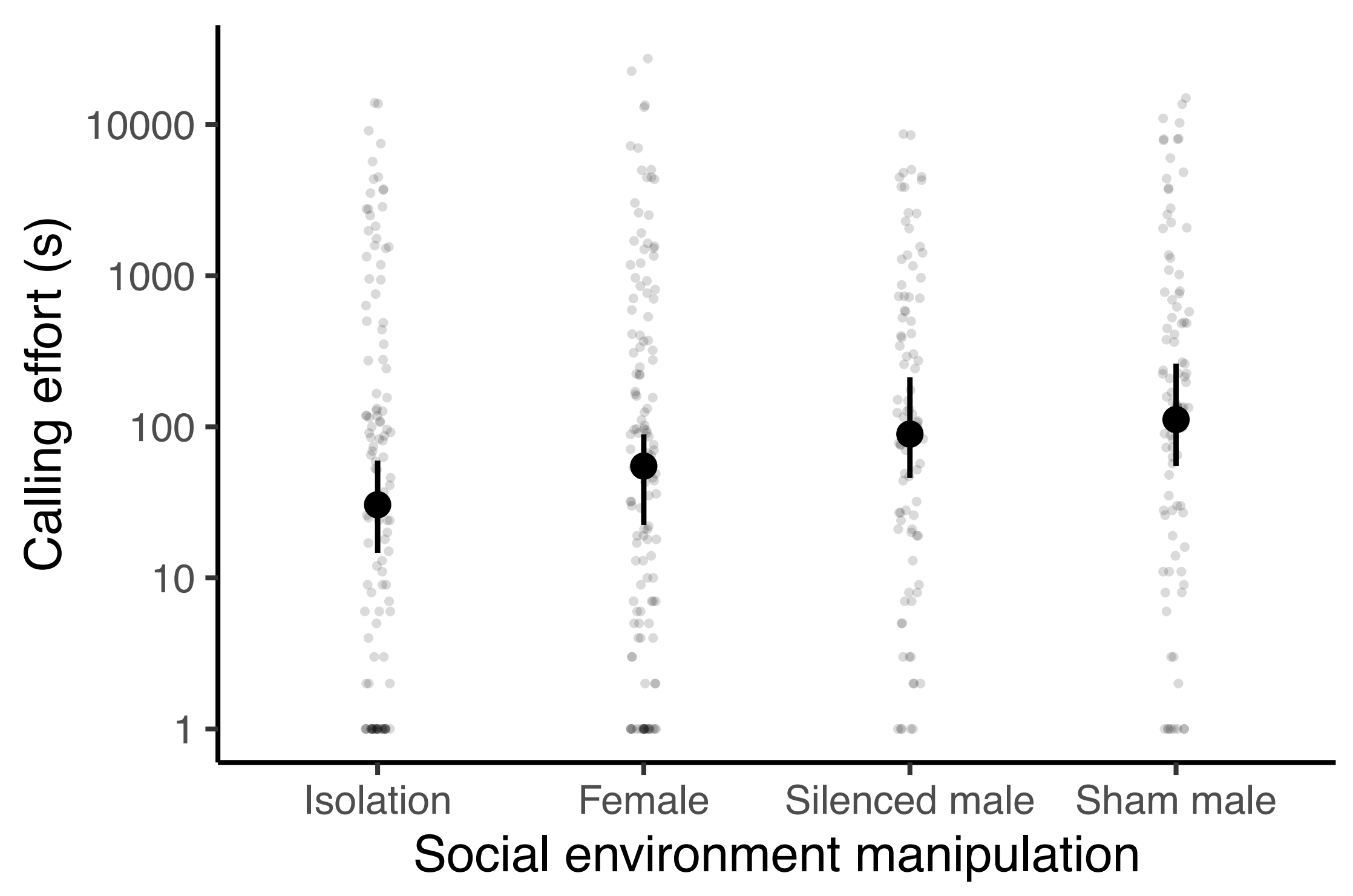




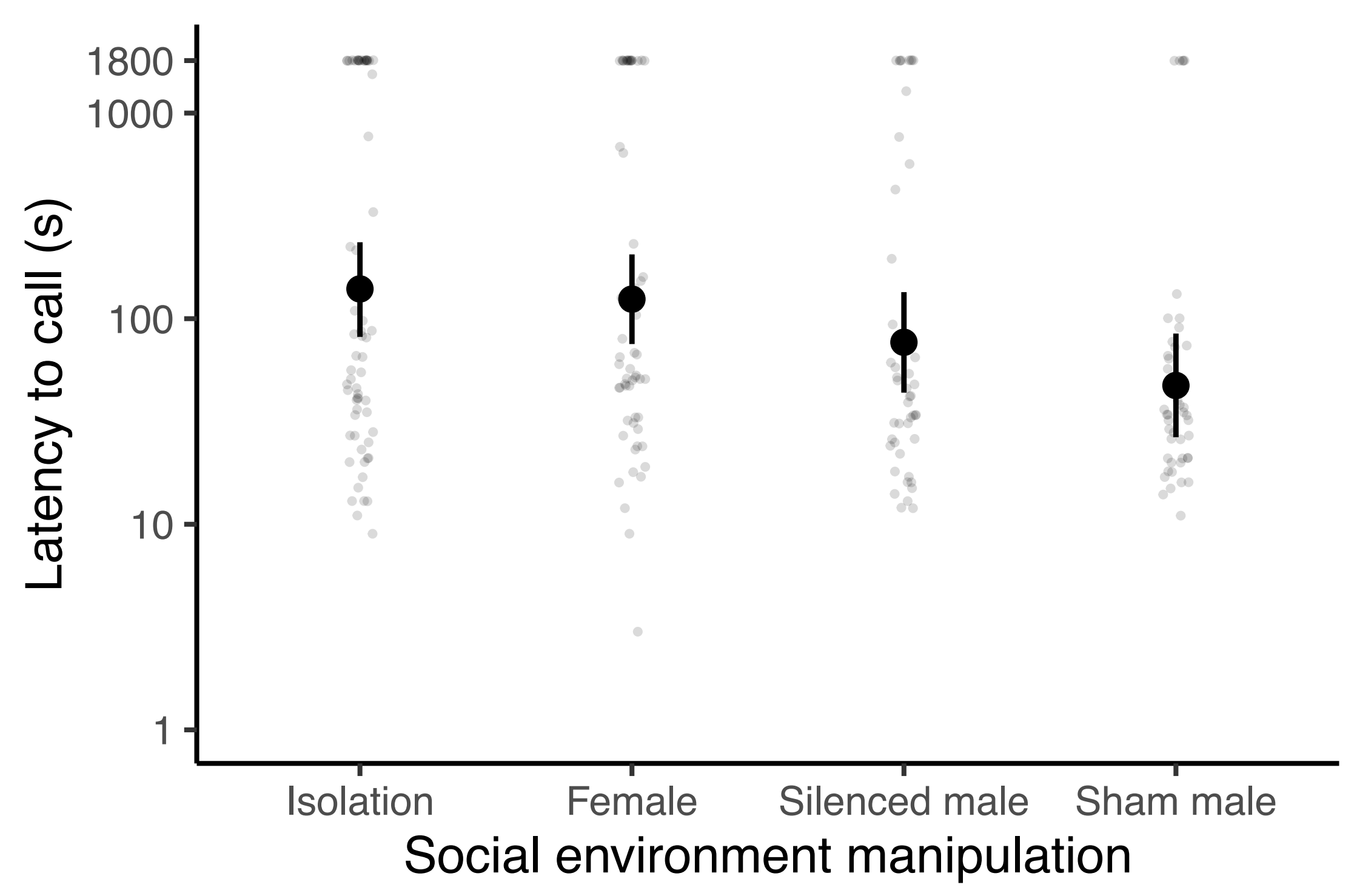




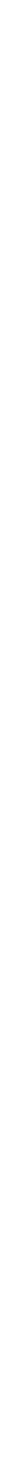

